\title{
Provision of hearing aids: Does specialist assessment cause delay?
}

\author{
Carl Watson, John A Crowther
}

\begin{abstract}
Objective-To identify the main delay in the provision of hearing aids for people with impaired hearing and identify possible problems and shortcomings caused by a community based hearing aid dispensing service.

Design-Prospective cohort analysis based on data collected from patients on the duration of hearing impairment, from the referral letters in respect of the general practitioners' findings on otoscopy, and from the ear, nose, and throat assessment in the clinic with respect to the outcome of specialist otoscopy and management of the hearing impairment.
\end{abstract}

Setting-General ear, nose, and throat outpatient clinic.

Patients-100 Consecutive patients aged 19-94 referred by general practitioners for the provision of hearing aids or for assessment and treatment of impaired hearing.

Results-Most patients with impaired hearing did not seek medical advice for at least a year. The time from referral by the general practitioner to the provision of a hearing aid was under two months. General practitioners consistently recognised normality on otoscopy but failed to recognise abnormality in eight of $\mathbf{4 5}$ cases. Seven patients required further investigation to exclude serious disease and nine had conditions amenable to surgery.

Conclusions - The main cause of delay in treating impaired hearing is failure by patients to seek help promptly. Specialist assessment of patients with impaired hearing is preferable and does not necessarily cause delay in providing hearing aids. The provision of hearing aids should remain a hospital based service.

\section{Introduction}

There are estimated to be 3.9 million adults in the United Kingdom with impaired hearing which might be helped by a hearing aid, ${ }^{1}$ and with an aging population the potential demand will inevitably increase. The arrangement for the provision of hearing aids within the NHS is almost exclusively by referral to an ear, nose, and throat outpatient clinic. As with many hospital based services at present lengthy delays may be encountered in certain areas. The Royal National Institute for the Deaf has recently challenged the need for hospital referral of all adults with hearing impairment, and in a recent publication, Hearing Aids-The Case for Change, it makes various proposals for a complete change in the provision of hearing aids in both the public and private sectors.

At the heart of the proposals is the creation of a new grade of technician, designated a hearing aid dispenser. The dispenser would be based in health centres and larger general practices accepting referrals directly from the general practitioner and undertaking audiometry, provision of hearing aids, and aftercare. If these changes were implemented inevitably general practitioners would have to take on greater responsibility for screening patients with hearing impairment. Before making any radical changes to the present system the potential problems created by these changes should carefully be considered.

This study aimed at identifying the main source of delay in providing hearing aids, assessing the accuracy of otoscopy performed by general practitioners, and identifying the proportion of patients with impaired hearing who require more than simple screening and provision of hearing aids.

\section{Subjects and methods}

Information was collected prospectively from 100 consecutive new patients with impaired hearing referred to the ear, nose, and throat outpatient clinic at this hospital. The patients were aged 18 or over and were referred by their general practitioner either specifically for the provision of a hearing aid or for assessment and treatment of their condition. For the purposes of analysis the two groups were kept separate. The Royal National Institute for the Deaf has recommended that under the new proposals patients with additional symptoms-for example, otorrhoeawould still warrant specialist referral; these referrals were excluded from the study.

All patients were seen in the clinic by consultants, senior registrars, or registrars. A questionnaire was used to record the following information: $(a)$ (from the referral letter) the reason for and date of referral and the general practitioner's findings on otoscopy; $(b)$ (from the patient) the duration of hearing impairment; $(c)$ the specialist's findings on otoscopy; $(d)$ the subsequent management, including need for wax removal, treatment of inflammatory ear disease, further investigations to exlude serious underlying disease, identification of surgically correctable impairment, and whether providing a hearing aid was appropriate.

Otoscopic findings in the clinic were compared with those recorded in the general practitioner's referral letter. It may be difficult to describe otoscopic findings precisely and we therefore graded these simply as normal or abnormal. Thus though a general practitioner's findings of abnormality may not have been in complete accordance with those made in the specialist clinic, they were considered to be correct or in agreement provided that some abnormality was described. Simple comparisons of interobserver agreement are misleading, as a proportion may be due to chance. Hence the kappa $(x)$ statistic was used. The details of $x$ have been described in similar studies ${ }^{23}$; in brief, $x$ ranges from -1 (complete disagreement), through 0 (no agreement), to 1 (complete agreement).

\section{Results}

The 100 consecutive new patients referred for impaired hearing accounted for roughly one in five of 
all new patients seen during the study period. Fifty four of the patients (mean age 71 (SD 13) years) were referred specifically for the provision of a hearing aid and 46 (mean age 55 (16)) for general assessment and treatment.

Delay in provision of hearing aid-The duration of impaired hearing before patients sought advice varied from less than six months to over 10 years (table I). In most cases the general practitioner made an immediate referral to the specialist clinic; in eight cases (four patients in each group), however, there was a delay of over six months before referral. The mean waiting time for patients to be seen in the ear, nose, and throat clinic was three weeks for both groups. For those needing a hearing aid an ear mould impression was taken during the initial visit. The delay thereafter - that is, until the completed mould was ready and the patient seen for fitting and instruction in the use of the aid - was about four weeks.

Otoscopy - In 55 cases the referral letter made no mention of the general practitioner's otoscopic findings. Among patients referred specifically for the provision of a hearing aid otoscopic findings were noted in 21 letters $(39 \%)$, and among patients referred for assessment these were noted in 24 letters $(52 \%)$. Table II shows the agreement between the general practitioners' and specialists' findings on otoscopy.

TABLE I - Duration of hearing impairment before referral. Figures are numbers of patients

\begin{tabular}{lccccccc}
\hline & \multicolumn{7}{c}{ Duration (years) } \\
\cline { 2 - 6 } & $<0 \cdot 5$ & $0 \cdot 5-1$ & -5 & -10 & $>10$ & Total \\
\hline Referral for hearing aid & 3 & 5 & 31 & 5 & 10 & 54 \\
Referral for assessment & 6 & 7 & 24 & 3 & 6 & 46 \\
\hline Total & 9 & 12 & 55 & 8 & 16 & 100
\end{tabular}

TABLE II-Otoscopic findings in groups referred for provision of hearing aid and for assessment. Figures are numbers of patients

\begin{tabular}{|c|c|c|c|}
\hline \multirow{2}{*}{$\begin{array}{l}\text { Specialists' } \\
\text { findings on } \\
\text { otoscopy }\end{array}$} & \multicolumn{2}{|c|}{ General practitioners' findings on otoscopy } & \multirow[b]{2}{*}{ Total } \\
\hline & Normal & Abnormal & \\
\hline & \multicolumn{2}{|c|}{ Group referred for hearing aid ${ }^{\star}$} & \\
\hline Normal & 13 & 0 & 13 \\
\hline Abnormal & 5 & 3 & 8 \\
\hline \multirow[t]{2}{*}{ Total } & 18 & 3 & 21 \\
\hline & \multicolumn{2}{|c|}{ Group referred for assessment $\dagger$} & \\
\hline \multirow{2}{*}{$\begin{array}{l}\text { Normal } \\
\text { Abnormal }\end{array}$} & 13 & 0 & 13 \\
\hline & 3 & 8 & 11 \\
\hline Total & 16 & 8 & 24 \\
\hline
\end{tabular}

General practitioners consistently recognised normality but failed to recognise abnormality in eight cases $(17 \cdot 8 \%)$. The most common abnormalities missed were perforation of the tympanic membrane, retraction of the tympanic membrane, tympanosclerosis, and impacted wax.

Management - Thirty patients needed removal of ear wax. Of the 54 patients referred specifically for a hearing aid and the 46 referred for general assessment, $46(85 \%)$ and $23(50 \%)$ respectively were fitted with an aid. Hearing impairment amenable to surgery was identified in three patients referred for a hearing aid and six patients referred for assessment. The surgically treatable causes of hearing impairment in these patients included otosclerosis, glue ear, and tympanic membrane perforation.

\section{Discussion}

The Royal National Institute for the Deaf has drawn attention to the magnitude of the problem of impaired hearing. According to the Office of Population Censuses and Surveys it is the second most common disability. ${ }^{1}$ Hearing impairment leads to communication difficulties and social isolation and may eventually cause depression. These effects may be avoided by early identification and treatment of the hearing loss. We broadly agree with the aims of the Royal National Institute's proposals - that is, the early diagnosis and treatment of hearing impairment-but disagree on how this would best be achieved.

In order to have the greatest impact on reducing delay in the provision of hearing aids it is essential to identify the factor causing most delay. In this study of 100 patients 79 had been aware of hearing impairment for over a year and 24 for over five years before seeking treatment. The waiting time for outpatient appointments is fortunately short in this department, and the average time between referral by the general practitioner and fitting a hearing aid is less than two months. The main delay in providing treatment therefore rested with the patients themselves, and only greater public awareness will encourage patients to present earlier. Undoubtedly outpatient waiting times are considerably longer in certain areas.

A survey by the Royal National Institute for the Deaf in 1984 suggested that the average waiting time for an ear, nose, and throat outpatient appointment was 16 weeks in the United Kingdom overall and 9.5 weeks in Scotland. ${ }^{4}$ There was considerable variation in waiting times, ranging from one to 132 weeks. The Scottish Home and Health Department (Edinburgh) and the Department of Health (London) were contacted to obtain more recent figures. The current waiting time for an ear, nose, and throat outpatient appointment in Scotland varies from two to 46 weeks. It is not possible to give a median waiting time from the figures available. The Department of Health denied keeping any information on outpatient waiting times, so that it is not possible to obtain similar information for England. The reasons for the considerable variation in outpatient waiting times for new referrals are complex, but include funding, staffing, facilities, referral patterns, and fluctuations in the local catchment population.

It is not clear whether delays in the provision of hearing aids are due solely to the size of outpatient waiting lists or to the shortage of audiological technicians. Central Nottinghamshire Health Authority has recently reduced the delay in obtaining an aid by 13 months by appointing an additional technician and providing funding for overtime working. ${ }^{5}$ The shortage of technicians may limit the number of patients with impaired hearing who may be seen in a clinic and leads in some areas to the policy of producing a secondary waiting list for a subsequent visit during which an ear mould impression is taken by a technician. This is unsatisfactory both for the patient and for the department because technicians' time is wasted in arranging further appointments, and if the delay in reattendance is lengthy the patient may require further removal of wax and yet another visit. Taking ear mould impressions on the first visit helps to minimise delay in this department, and the adoption of this policy is a factor claimed for the improved service in central Nottinghamshire.

There are estimated to be 150 unfilled vacancies for audiological technicians in the NHS. The current problems of recruiting hospital technicians suggest that difficulties would also be encountered in filling vacancies for hearing aid dispensers, and any attempt to make these posts more attractive to applicants could lead to further attrition of the hospital service. This being the case, funding should be utilised to increase the number of audiological technicians rather than to introduce the new grade of hearing aid dispenser. 
Hearing loss is not a diagnosis; rather it is a symptom, and patients require careful assessment. The Royal National Institute for the Deaf proposes that hearing aid dispensers should perform this function. Failure to recognise and further investigate asymmetric sensorineural hearing loss or unilateral glue ear may lead to neglect of serious disease such as acoustic neuroma or postnasal carcinoma. Though the incidence of such diseases is low, even the small sample of 100 patients in this study included seven who warranted further investigation to exclude such conditions. Recognising glue ear and other middle ear abnormalities, which may be amenable to surgical correction, requires skill in otoscopy. Our results suggest that otoscopy performed by general practitioners may miss abnormalities in a considerable number of patients. As a result of inadequate assessment some patients may be denied the option of surgical treatment.

Comparison of the group referred specifically for the provision of a hearing aid with the group referred for assessment suggests that general practitioners already perform some screening and in many cases assess their patients' needs correctly. The group referred for a hearing aid contained a higher proportion of patients who required an aid (46/54) compared with those referred for assessment (23/46). Similarly a greater proportion of patients referred for assessment had hearing loss amenable to surgery. The kappa values for interobserver agreement (table II), however, were substantially different for each group, showing much poorer agreement in the group referred for hearing aids. When viewed alongside the age distribution of patients in each group the findings suggest that any screening which is presently being done is based predominantly on the patient's age rather than clinical assessment.

We do not seek to criticise general practitioners in this regard; many will have had little or no formal training in otoscopy since undergraduate days. A recent survey showed that roughly one sixth of vocational training schemes included ear, nose, and throat attachments, and only a quarter of these included full time six month posts. ${ }^{6}$ For the Royal National Institute for the Deaf to suggest that general practitioners wishing to provide hearing aid services based in their own practices might receive "supplementary training which could be organised during a single or two days" is frankly naive. The suggestion that screening should be performed by the dispenser is unacceptable, and were general practitioners to delegate in this manner they would almost certainly carry ultimate legal responsibility.

\footnotetext{
1 Royal National Institute for the Deaf. Hearing aids-the case for change London: RNID, 1988

2 Spitzer RL, Cohen J, Fleiss JL. Endicott J. Quantification of agreement in psychiatric diagnosis. Arch Gen Psychiatry 1967;17:83-7.

Hardcastle PF, von Haacke N, Murray JAM. Observer variation in clinical examination of the nasal airway. Clin Otolaryngol 1984:10:3-7.

Johnson J, Grover B, Martin MA A surev of National Health Serice heuring aid services. London: Roval National Institute for the Deaf, 1984.

sercices. London. Royal National Institute for the Deaf, 1984. 1989:9:(18):6.

Rivron RP, Clayton MI. Ear, nose, and throat teaching. Clin Otolaryngol 1988:13:133-8.
}

(Accepted 30 May 1989)

\title{
Lesson of the Week
}

\section{Multiple forms of epileptic attack secondary to a small chronic subdural haematoma}

\author{
S C Jones, J M Bamford, J Heath, N Bradey, R V Heatley
}

Comparatively rare manifestations of epilepsy may be the presenting features of a subdural haematoma

St James's University Hospital, Leeds LS9 7TF $\mathrm{S} C$ Jones, MRCP, registrar in medicine

J M Bamford, MRCP, senior registrar in neurology

J Heath, MRCP, senior registrar in clinical neurophysiology

N Bradey, MRCP, senior registrar in neuroradiology R V Heatley, MRCP, senior lecturer in medicine

Correspondence to: Dr S C Jones, Pinderfields General Hospital, Wakefield

WF1 4DG.

Br.Med f 1989;299:439-41
Since the introduction of computed tomography (CT) small or asymptomatic chronic subdural haematomas have been recognised with increasing frequency, though their detection may depend on the use of contrast enhancing agents.' Patients with chronic subdural haematoma commonly present with headache, disturbances of higher cerebral function such as personality change and confusion, or features of raised intracranial pressure. Hemiparesis, with dysphasia if the lesion is over the dominant hemisphere, is the most frequent focal neurological deficit. Epilepsy is traditionally thought of as a rare presenting feature. ${ }^{2}$ We describe a patient in whom several different forms of epilepsy were the only clinical manifestations of a very small chronic subdural haematoma.

\section{Case history}

A 75 year old man was admitted after a witnessed tonic-clonic convulsion. Over the previous two weeks he had suffered three episodes of sudden, complete inability to speak, during which he remained fully conscious without any abnormal movements being noted. Each episode lasted about five minutes. He was seen by his general practitioner, who diagnosed transient ischaemic attacks but did not prescribe aspirin. The patient denied headache, and neither he nor his family could recall any recent head injury. He was taking chlorpropamide for maturity onset diabetes but there was no previous history of epilepsy or cerebrovascular disease. He had never had an excessive alcohol intake. Full neurological examination on admission showed nothing abnormal, and in particular there was no disturbance of higher cerebral function. Blood glucose, electrolyte, and calcium concentrations remained normal throughout.

Over the next 24 hours attacks were witnessed during which the patient would become vacant and his head and eyes would deviate to the right. His right arm would then become elevated with external rotation of the shoulder followed by alternating flexion and extension at the elbow while the wrist remained flexed and the fingers extended and abducted. These attacks would last between five and 60 seconds and were followed by a variable period of confusion. They could occur several times an hour, often without complete recovery of consciousness in between. During periods of full consciousness, however, no neurological defici could be detected. An attack could be terminated with intravenous diazepam, which would continue to act for about 30 minutes. He was given a loading dose of intravenous phenytoin followed by conventional oral doses.

About 48 hours after admission a further change was noted, in that he was continuously confused with a fluctuating level of consciousness. On occasions he 